\title{
Purification and many-body localization in cold atomic gases
}

\author{
Felix Andraschko, ${ }^{1,2}$ Tilman Enss, ${ }^{3}$ and Jesko Sirker ${ }^{1,2}$ \\ ${ }^{1}$ Department of Physics and Research Center OPTIMAS, \\ Technical University Kaiserslautern, D-67663 Kaiserslautern, Germany \\ ${ }^{2}$ Department of Physics and Astronomy, University of Manitoba, Winnipeg, Canada R3T 2N2 \\ ${ }^{3}$ Institut für Theoretische Physik, Universität Heidelberg, D-69120 Heidelberg, Germany
}

\begin{abstract}
We propose to observe many-body localization in cold atomic gases by realizing a Bose-Hubbard chain with binary disorder and studying its non-equilibrium dynamics. In particular, we show that measuring the difference in occupation between even and odd sites, starting from a prepared densitywave state, provides clear signatures of localization. As hallmarks of the many-body localized phase we confirm, furthermore, a logarithmic increase of the entanglement entropy in time and Poissonian level statistics. Our numerical density-matrix renormalization group calculations for infinite system size are based on a purification approach which allows to perform the disorder average exactly, thus producing data without any statistical noise, and with maximal simulation times of up to a factor 10 longer than in the clean case.
\end{abstract}

Cold atomic gases with or without optical lattices are an ideal platform to realize model Hamiltonians of strongly correlated quantum systems by offering an unprecedented control over the microscopic parameters [1]. Among the many achievements are the observation of the superfluid to Mott insulator transition for a Bose gas held in a three-dimensional optical lattice [2], the realization of the Tonks-Girardeau regime in a quasi onedimensional (1D) Bose gas [3, and the simulation of the non-equilibrium dynamics in an almost integrable onedimensional quantum system [4].

While these experiments have all been performed on very clean systems, there is also a tremendeous interest in building quantum simulators for models with disorder. This interest is sparked by the unavoidable presence of disorder and impurities in real materials which can lead to completely new physics, such as the Kondo effect and Anderson localization 5. For cold atomic gases there have been several different approaches to realize disorder. The first experiments have employed quasiperiodic lattices or laser speckles to study Anderson localization in effectively non-interacting Bose condensates in one and three dimensions [6]. As an alternative it has been suggested to use the repulsive interactions between two different species of atoms - with one being effectively immobile - to obtain a binary disorder potential for the mobile species on the scale of the lattice constant [7, 8. Experimentally, the localization of bosonic atoms by fermionic impurities has been demonstrated 9 .

Theoretically, it has been suggested by Anderson [5. that a localized phase might be stable against small interactions, a result which has been supported by a recent study [10] leading to a renewed interest in many-body localization (MBL). In interacting spin chains with a random magnetic field drawn from a box distribution, in particular, a transition is found between a delocalized, ergodic phase at weak disorder and a many-body localized (MBL) phase at strong disorder [11-13]. In an MBL phase, where all many-body eigenstates are localized, a simple picture emerges: In this case one can separate a chain 14 into segments of length $\ell \gg \xi$ where $\xi$ is the localization length. The many-body eigenstates of the whole chain are then, to a good approximation, product states of the eigenstates in each segment. Importantly, projectors onto the eigenstates of a segment are local conserved charges with finite support and have to be included in a generalized Gibbs ensemble 15. This constrains the dynamics and prevents thermalization in any subsystem [16, 17. Residual interactions between segments make these charges quasi-local, i.e., contributions to the conserved charge with spatial support on any length scale $r$ exist, but are suppressed as $\exp (-r)$. The conserved charges thus remain relevant for transport and non-equilibrium dynamics [18. The residual long-range interactions are also responsible for the observed growth of the entanglement entropy, $S \sim \ln t$, with time $t$ if the system is prepared in a product state and evolves in time [16, 19, 20]. This is one of the hallmarks of an MBL phase, in contrast to the linear growth in systems without disorder 21 and the extremely slow increase, $S \sim \ln \ln t$, found for a noninteracting model with bond disorder [22]. Experimentally, however, this new state of matter has not yet been detected and the question which kind of system and which local observables are appropriate for this purpose is considered as one of the main open problems in this field 23].

In this letter we discuss the possible realization and observation of many-body localization in a system of cold atoms. Consider two interacting species of bosons in an optical lattice, with one of them frozen to form a binary disorder potential for the other, mobile species [7, 8]. The effective Hamiltonian for the mobile bosons is

$$
\begin{aligned}
H & =-\frac{J}{2} \sum_{j}\left(a_{j}^{\dagger} a_{j+1}+h . c .\right) \\
& +\sum_{j}\left[U n_{j}\left(n_{j}-1\right) / 2+V n_{j} n_{j+1}+J D_{j} n_{j}\right],
\end{aligned}
$$


where $J$ is the hopping amplitude, $n_{j}=a_{j}^{\dagger} a_{j}$ the local density, $U$ the onsite, and $V$ the nearest-neighbor interaction. The effective binary disorder potential $D_{j}$ is drawn randomly according to $D_{j}= \pm D$, and we have neglected the trapping potential. We consider in the following the case where the mobile species is prepared in the initial density-wave state $\left|\Psi_{0}\right\rangle=|010101 \cdots\rangle$. During the ensuing time evolution under the Hamiltonian (1) we propose to measure the difference in occupation between the even and odd sites, $\Delta n=N^{-1} \sum_{j}(-1)^{j}\left(\left\langle n_{j}\right\rangle-1 / 2\right)$, where $N$ is the number of lattice sites. Exactly this setup has already been realized in the clean case, i.e., for a single species of bosons [24. By freezing the immobile bosons into a quantum state which is close to an equal superposition of Fock states $\left|n_{1}, n_{2}, \cdots\right\rangle$ with $n_{j} \in\{0,1\}$, the time evolved state is automatically averaged over all binary disorder configurations $[8,25$. This purification method can also be used for numerical computations and is explained in detail below. Our proposal thus combines realizing disorder in optical lattices using two species of atoms [9] with techniques to prepare initial states and to measure their non-equilibrium dynamics 24]. Experimentally, this can be realized, for instance, with two hyperfine states of ${ }^{87} \mathrm{Rb}$ atoms loaded into a state-dependent optical lattice: the wavelength controls the relative hopping amplitude of both states, while the intraspecies interaction is tuned by a Feshbach resonance, and the interspecies interaction depends on the intensity of the laser beam. In this way, both the ratio of onsite interaction $U$ to hopping $J$ and the coupling between mobile and disorder atoms can be tuned independently, and bimodal disorder with very short-range correlations is realized [8.

Let us first discuss the cases $V=0$ with $U=0$ or $U=\infty$ with strong disorder $D \gg 1$. In this case, each disorder configuration splits the chain into segments of equal potential $D_{i}$, which communicate very little with their neighboring segments due to the mismatch of the local potential energy and the kinetic energy, $J D \gg J$ : for a given segment, $\Delta n$ has contributions from neighboring segments of the order of $1 / D^{2}$. Thus, the segments become isolated in the limit $D \rightarrow \infty$. The time evolution of the whole chain is then given by summing up the independent time evolution of open segments $\Delta n^{(\ell)}(t)$ of varying length $\ell$, weighted by their probability of occurrence, $p_{\ell}=\ell / 2^{\ell+1}$ with $\sum_{\ell} p_{\ell}=1$, leading to $\Delta n^{D=\infty}(t)=$ $\sum_{\ell=1}^{\infty} p_{\ell} \Delta n^{(\ell)}(t)$ both for $U=0$ and $U=\infty$ [26]. It is easy to see that only segments of odd chain length contribute to the long-time average with $\overline{\Delta n}_{\text {odd }}^{(\ell)}=1 /(2 \ell)$, so that $\overline{\Delta n}=\sum_{\ell} p_{\ell} \overline{\Delta n}^{(\ell)}=\frac{1}{2} \sum_{\ell \text { odd }} p_{\ell} / \ell=\frac{1}{6}$.

Fig. 1(a) shows the time evolution in the clean case, where for both $U=0$ and $U=\infty$ one finds $\Delta n^{D=0}(t)=$ $J_{0}(2 J t) / 2$, with the Bessel function $J_{0}(x)$ 27, 28. In comparison, the time evolution in the strongly disordered case, Fig. 1.(b), appears very complicated. Yet, including only segments up to a maximum length $L$ already gives an approximation with an exponentially small error $\sim$ $L / 2^{L}$ at all times, because $p_{\ell}$ decays exponentially.

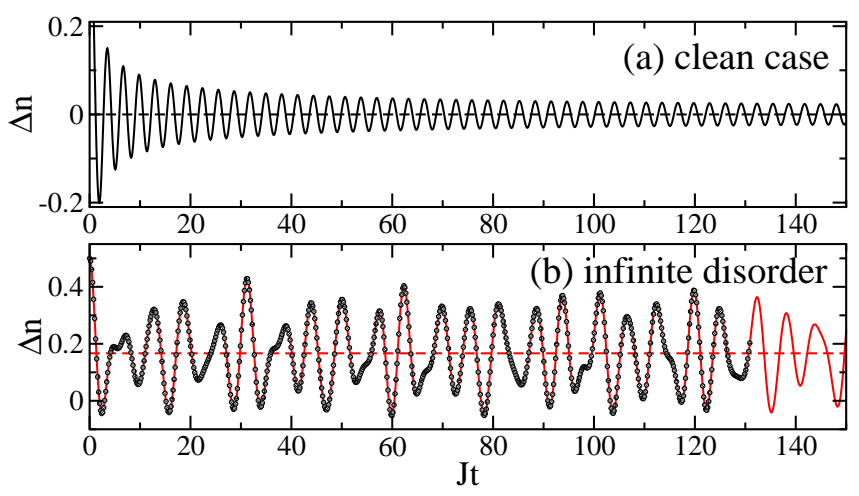

FIG. 1: (Color online) $\Delta n(t)$ for model (1) with $V=0$ and $U=0$ or $U=\infty$ : (a) $\Delta n^{D=0}$ vs (b) $\Delta n^{D=\infty}$ (solid red) with average $\overline{\Delta n}=1 / 6$ (dashed red); symbols are LCRG results.

The model (1) is difficult to treat numerically because of the unrestricted local Hilbert space dimension for finite interaction strength $U$. We will therefore first concentrate on the limit $U \rightarrow \infty$, where numerical methods are very efficient. We will firmly establish that an MBL phase exists in this limit before returning to the case of finite $U$ at the end of this letter. For $U \rightarrow \infty$, the above model maps onto the spin- $1 / 2 X X Z$ chain

$$
H=-J \sum_{i}\left(s_{i}^{x} s_{i+1}^{x}+s_{i}^{y} s_{i+1}^{y}-\Delta s_{i}^{z} s_{i+1}^{z}-D_{i} s_{i}^{z}\right),
$$

with anisotropy $J \Delta=V\left[29 .\left|\Psi_{0}\right\rangle\right.$ is then the Néel state and $\Delta n=N^{-1} \sum_{j}(-1)^{j}\left\langle s_{j}^{z}\right\rangle$ is the staggered magnetization. For $\Delta=0$, the dynamics is again given by Fig. 1(a) in the clean case and by Fig. 1 (b) in the strongly disordered case. For alkali atoms, $V \ll J$, so that a realization of the $X X Z$ model with substantial $\Delta$ is not easily achievable. We note, though, that the isotropic Heisenberg chain, $\Delta=1$, has recently been realized using two boson species, and that the non-equilibrium dynamics has been studied with single-site addressability 30. Furthermore, longer-range interactions are also present if dipolar gases or polar molecules are used [31].

To simulate the non-equilibrium dynamics of the BoseHubbard model (1) and the $X X Z$ model (2) for all interaction and disorder strengths and to perform an exact disorder average we use the light cone renormalization group (LCRG) [28]. The LCRG algorithm is a variant of the density-matrix renormalization group (DMRG) technique [32] based on the Lieb-Robinson bounds [33]: a local measurement at time $t$ is affected only by the degrees of freedom within its light cone. The opening angle of the light cone, or spreading velocity, is determined by model parameters. For lattice models with short-range interactions, the time evolution operator $\mathcal{U}=\exp (-i H t)$ has a 
Trotter-Suzuki decomposition with a checkerboard structure: within the LCRG, a light cone out of the infinite checkerboard is sufficient to compute the time evolution of local observables in an infinite system [28, 34].

To treat disorder, one straightforward possibility for a finite system is to compute the time evolution for one particular disorder configuration, and then repeat the calculation for many different configurations to obtain the disorder average. Here, we instead use purification for an infinite system in order to perform the full disorder average in a single run [25], at the expense of enlarging the Hilbert space. Specifically, for the $X X Z$ chain, an ancilla spin- $1 / 2, \vec{s}_{i \text {,anc }}$, is added to each lattice site with an Ising coupling, $D_{i} s_{i}^{z} \mapsto 2 D s_{i}^{z} s_{i \text {,anc }}^{z}$. The state of $s_{i \text {,anc }}^{z}= \pm 1 / 2$ now determines the local Zeeman field $D_{i}= \pm D$. There is no coupling between different ancilla spins, hence they have no dynamics and represent static disorder. The time evolution of the disorder average is given by the evolution from a prepared product state $\left|\psi_{0}\right\rangle \otimes \mid$ dis $\rangle$ in the enlarged Hilbert space of spins and ancillas, where $\mid$ dis $\rangle=\bigotimes_{j}\left(|\uparrow\rangle_{j}+|\downarrow\rangle_{j}\right) / \sqrt{2}$ is the fully mixed state for the ancillas. The disorder averaged expectation value of an operator $O$ is then obtained by measuring the expectation value of the operator $O \otimes \mathbb{1}_{\text {anc }}$ in the enlarged Hilbert space. Although the local Hilbert space dimension is doubled, the LCRG algorithm works even more efficiently for strongly disordered systems than for clean systems, and real times up to $J t \sim 100$ are reached in our simulations, where we keep the truncation error in each renormalization group step smaller than $10^{-8}$ by dynamically increasing the number of kept states up to 20000 . Responsible for these long simulation times is the slow logarithmic growth of the entanglement entropy, $S_{\text {ent }}$, for $\Delta \neq 0$, see Fig. 2. Here, $S_{\text {ent }}=-\operatorname{Tr} \rho_{B} \ln \rho_{B}$, where $\rho_{B}$

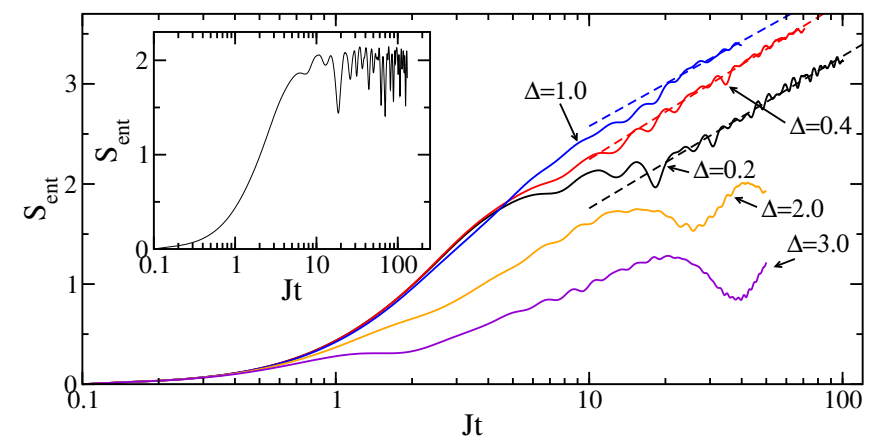

FIG. 2: (Color online) $S_{\text {ent }}$ for the $X X Z$ chain 2 in the strongly disordered case $D=4000$. For small $\Delta$ we find asymptotically $S_{\text {ent }}(t) \sim \ln t$ (dashed lines are fits for $t>20$ ). Inset: $S_{\text {ent }}(t)$ saturates for $\Delta=0$ and infinite disorder.

is the reduced density matrix obtained by cutting the infinite chain, $A \otimes B$, of spins and ancillas in half. Since entanglement in the static ancillas is mediated by the spins, $S_{\text {ent }}$ has the same functional dependence on time as the disorder averaged entanglement entropy of a spin- only system [26]. The logarithmic increase for $\Delta \neq 0$ is the same behavior as seen for the $X X Z$ model with the magnetic fields $D_{i}$ drawn from a box distribution [20, and is a hallmark of an MBL phase. On the other hand, $S_{\text {ent }}$ saturates for $\Delta=0$ and infinite binary disorder, see the inset of Fig. 2. The latter behavior can be easily understood by noting that $S_{\text {ent }}$ for a block of size $n \leq \ell$ of a finite chain segment of spins and ancillas with length $\ell$ is bounded, $S_{\text {ent }} \leq n \ln 4$. Since $p_{\ell}$ decreases exponentially, a strict bound for $S_{\text {ent }}$ at all times exists [26]. This is different from the case of strong bond disorder, where $S_{\text {ent }} \sim \ln \ln t[22$.

In Fig. 3(a,b) we show $\Delta n(t)$ for strong and intermediate disorder. In all cases shown, $\Delta n(t)$ does not decay
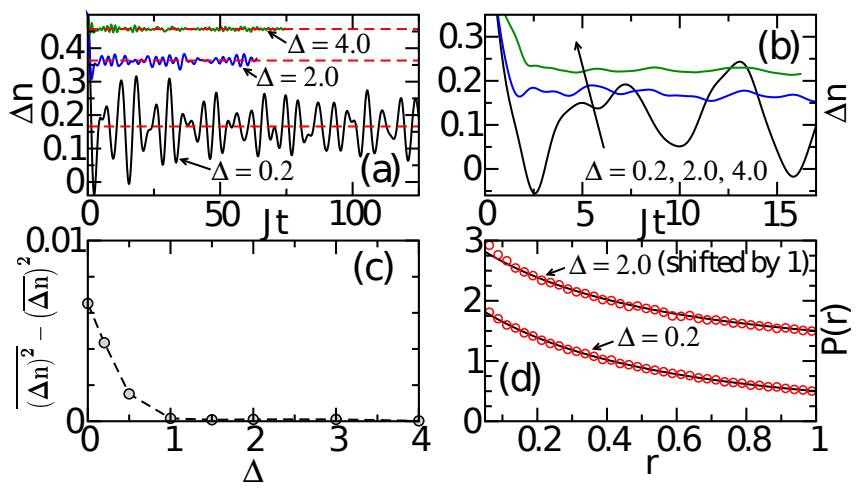

FIG. 3: (Color online) $X X Z$ chain: (a) $\Delta n(t)$ for $D=4000$ with averages (dashed lines). $D=1.5$ : (b) $\Delta n(t)$, (c) variance of $\Delta n(t)$ for $t>5$, and (d) $P(r)$ for chains of length $N=14$ (symbols) and $P(r)=2 /(1+r)^{2}$ (solid lines).

to zero, indicating that the system does not thermalize. A clear experimental indication that localization in an interacting system is observed, is the strong reduction of the variance of $\Delta n(t)$ with increasing $\Delta$, see Fig. 3(c).

To further support our findings of an MBL phase for the $X X Z$ model with binary disorder, we have also calculated the level statistics for finite chains of up to $N=14$ sites in the $S^{z}=0$ sector by exact diagonalization of all $2^{N}$ possible disorder realizations. In the integrable $X X Z$ chain without disorder, a full set of local integrals of motion exists, which allows to completely classify the eigenvalues by the corresponding quantum numbers. The spectrum is therefore uncorrelated and the corresponding level statistics Poissonian, $\mathcal{P}(s)=\exp (-s)$, in terms of the level spacing $s$. Disorder breaks integrability, so that the level-spacing distribution, if the many-body states are extended, will follow a Wigner distribution, $\mathcal{P}(s)=(\pi s / 2) \exp \left(-\pi s^{2} / 4\right)$. This can also be understood as a crossover from integrability to quantum chaos [35. However, once localization sets in, the spectrum will again become uncorrelated, because localization creates new quasi-local conserved charges, leading to a Poissonian level statistics. In this case chaos is incomplete and the system keeps a memory of the initial state [35. If a 
critical $D_{c} \neq 0$ for localization exists, we therefore expect to go from a Poissonian ( $D=0$, integrable) to a Wigner distribution ( $0<D<D_{c}$, non-integrable and delocalized), and then again back to a Poissonian $\left(D>D_{c}\right.$, localized) [26]. Here, we concentrate on the regime far from the clean integrable limit. In order to avoid the ambiguous definition of an average gap based on a construction of a continuous density of states from finite size data, we consider the ratio $r$ between two consecutive gaps of adjacent energy levels as defined in Refs. [11, 12. If the level statistics is Poissonian, the distribution function of gap ratios $0 \leq r \leq 1$ is given by $P(r)=2 /(1+r)^{2}$. As shown in Fig. 3(d), this is in good agreement with the numerical results [26].

Of particular interest is the isotropic Heisenberg chain, $\Delta=1$, which has recently been realized in cold atomic gases 30] and approximately describes materials such as $\mathrm{Sr}_{2} \mathrm{CuO}_{3}$ and $\mathrm{SrCuO}_{2}$ 36. In the latter case, a doping with non-magnetic impurities, such as $\mathrm{Pd}$, is possible, which randomly replace the magnetic $\mathrm{Cu}^{2+}$ ions [37]. As in the model (2), for strong disorder, the chain then separates into segments, however, the coupling between the segment ends is now not an Ising, but a Heisenberg coupling caused by next-nearest neighbor interactions [38. In such systems, many-body localization should also occur, preventing local excitations from spreading. However, phonons complicate the observation of MBL, leaving cold atomic gases as a particularly clean and promising realization. In Fig. 4, results for the Heisenberg model with binary disorder are shown. Here, clear signa-

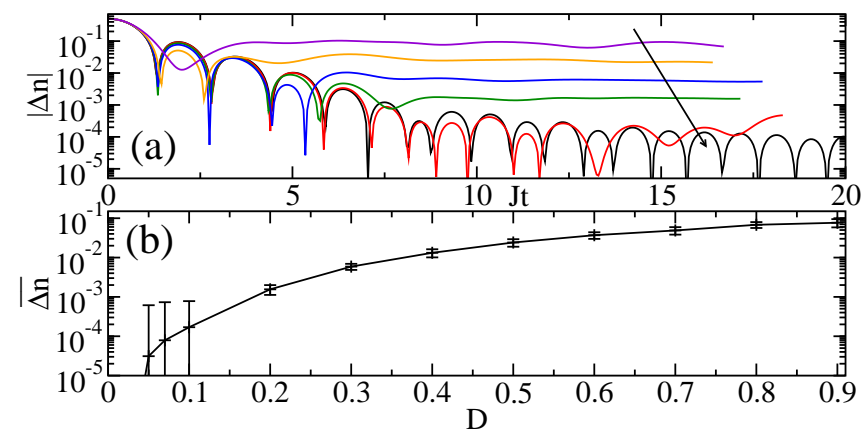

FIG. 4: (Color online) (a) $\Delta n(t)$ for the Heisenberg point, $\Delta=1$, and disorder strengths $D=0.9,0.5,0.3,0.2,0.1$, 0.0 (in arrow direction). (b) $\overline{\Delta n}$ obtained by averaging over intervals $\left[t_{\min }, 20\right]$ with $t_{\min }>5$ variable (see error bars).

tures for MBL are already seen for small disorder, with $\Delta n(t)$ quickly approaching a non-zero constant value, see Fig. 4(a), while $\Delta n(t)$ decays completely in the clean case [27. By averaging over different time intervals, we can extract an estimate for the long-time average $\overline{\Delta n}$ with error bounds, see Fig. 4(b). The results are consistent with a critical value $D_{c}$ for the localization transition which is either zero or finite, but with $D_{c}<0.2$. A detailed analysis of the phase diagram, including the crossover from integrable to (incomplete) chaotic behavior, will be presented elsewhere 39.

Let us finally return to the full Bose-Hubbard model (1), which we have proposed to realize experimentally. We concentrate on the case of vanishing nearest-neighbor interaction, $V=0$, realized in alkali atoms. For infinite disorder strength, the system still separates into decoupled chain segments for any interaction strength $U$. In this case, we can simulate the dynamics for arbitrary times by exactly diagonalizing the segments, see Fig. 5(a). Note that in the limit $D \rightarrow \infty, S_{\text {ent }}(t)$ is bounded: there is no many-body localization in this case. At finite disorder, we again use the LCRG algorithm to simulate the system. Results for $\Delta n(t)$ and $S_{\text {ent }}(t)$ are shown in Fig. 5(b,c), respectively. Although the simula-
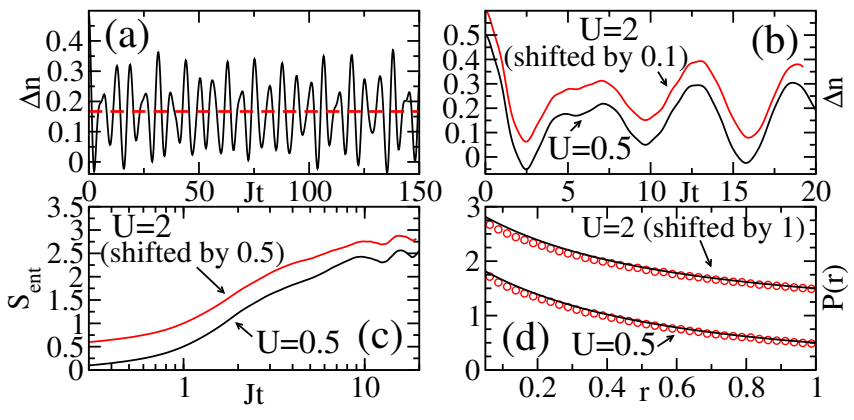

FIG. 5: (Color online) Bose-Hubbard model with $V=0$. (a) $\Delta n(t)$ for $D=\infty$ and $U=2$ with average (dashed line). Disorder strength $D=6$ : (b) $\Delta n(t)$, (c) $S_{\text {ent }}(t)$, and (d) $P(r)$ for $N=14$ (symbols) and $P(r)=2 /(1+r)^{2}$ (solid lines).

tion time is more limited than in the $X X Z$ case, $\Delta n(t)$ seems to remain nonzero, while the data for $S_{\text {ent }}$ are consistent with a logarithmic increase, as expected in an MBL phase. This is corroborated further by the distribution function of gap ratios $P(r)$, see Fig. 5 , consistent with a Poissonian level statistics.

To conclude, we have proposed to study many-body localization in cold atomic gases by realizing a BoseHubbard model with binary disorder provided by a second species, and studying its quench dynamics. Both experimentally as well as in numerical calculations one can make use of purification to achieve an automatic disorder average. By implementing the purification scheme into a DMRG algorithm, we have shown that the nonequilibrium dynamics can be simulated in a single run without any stochastic noise, and with simulation times for strong disorder which are significantly longer than in the clean case, making DMRG-type algorithms an ideal tool to investigate infinite, disordered systems. Both in the Bose-Hubbard model as well as in the $X X Z$ chain limit we have shown that an MBL phase exists and can be detected by measuring the one-point function $\Delta n(t)$.

F.A. and J.S. acknowledge support by the Collaborative Research Centre SFB/TR49, the Graduate School of Excellence MAINZ (DFG, Germany), as well as NSERC 
(Canada). We are grateful to the Regional Computing Center at the University of Kaiserslautern, the AHRP, and Compute Canada for providing computational resources and support.

[1] I. Bloch, J. Dalibard, and W. Zwerger, Rev. Mod. Phys. 80, 885 (2008).

[2] M. Greiner et al., Nature (London) 415, 39 (2002).

[3] B. Paredes et al., Nature (London) 429, 277 (2004).

[4] T. Kinoshita, T. Wenger, and D. S. Weiss, Nature (London) 440, 900 (2006).

[5] P. W. Anderson, Phys. Rev. 109, 1492 (1958).

[6] G. Roati et al., Nature (London) 453, 895 (2008); J. Billy et al., Nature (London) 453, 891 (2008); M. White et al., Phys. Rev. Lett. 102, 055301 (2009); F. Jendrzejewski et al., Nat. Phys. 8, 398 (2012).

[7] U. Gavish and Y. Castin, Phys. Rev. Lett. 95, 020401 (2005); T. Roscilde and J. I. Cirac, Phys. Rev. Lett. 98, 190402 (2007); S. Morrison et al., New J. Phys. 10, 073032 (2008).

[8] B. Horstmann, J. I. Cirac, and T. Roscilde, Phys. Rev. A 76, 043625 (2007); B. Horstmann, S. Dürr, and T. Roscilde, Phys. Rev. Lett. 105, 160402 (2010).

[9] S. Ospelkaus et al., Phys. Rev. Lett. 96, 180403 (2006).

[10] D. M. Basko, I. L. Aleiner, and B. L. Altshuler, Ann. Phys. (NY) 321, 1126 (2006).

[11] V. Oganesyan and D. A. Huse, Phys. Rev. B 75, 155111 (2007).

[12] A. Pal and D. A. Huse, Phys. Rev. B 82, 174411 (2010).

[13] C. Monthus and T. Garel, Phys. Rev. B 81, 134202 (2010); E. Canovi et al., Phys. Rev. B 83, 094431 (2011); J. Z. Imbrie, arXiv:1403.7837 T. Grover, arXiv:1405.1471.

[14] We concentrate here on the one-dimensional case but the argument is general and also applicable in higher dimensions.

[15] M. Rigol, V. Dunjko, V. Yurovsky, and M. Olshanii, Phys. Rev. Lett. 98, 050405 (2007).

[16] R. Vosk and E. Altman, Phys. Rev. Lett. 110, 067204 (2013).

[17] C. Gogolin, M. P. Müller, and J. Eisert, Phys. Rev. Lett. 106, 040401 (2011); G. Carleo, F. Becca, M. Schiró, and M. Fabrizio, Sci. Rep. 2, 243 (2012); M. Serbyn, Z. Papić, and D. A. Abanin, Phys. Rev. Lett. 111, 127201 (2013); D. A. Huse and V. Oganesyan, arXiv:1305.4915. Y. Bar Lev and D. R. Reichman, Phys. Rev. B 89, 220201(R) (2014); R. Vasseur, S. A. Parameswaran, and J. E. Moore, arXiv: 1407.4476.

[18] T. Prosen, Phys. Rev. Lett. 106, 217206 (2011); J. Sirker, N. P. Konstantinidis, F. Andraschko, and N. Sedlmayr, Phys. Rev. A 89, 042104 (2014).

[19] G. De Chiara, S. Montangero, P. Calabrese, and R. Fazio, J. Stat. Mech. P03001 (2006); M. Žnidarič, T. Prosen, and P. Prelovšek, Phys. Rev. B 77, 064426 (2008); R. Vosk and E. Altman, Phys. Rev. Lett. 112, 217204 (2014); A. Nanduri et al., Phys. Rev. B 90, 064201; M. Serbyn et al., arXiv:1403.0693

[20] J. H. Bardarson, F. Pollmann, and J. E. Moore, Phys. Rev. Lett. 109, 017202 (2012).

[21] S. Bravyi, M. B. Hastings, and F. Verstraete, Phys. Rev.
Lett. 97, 050401 (2006).

[22] F. Iglói, Z. Szatmári, and Y.-C. Lin, Phys. Rev. B 85, 094417 (2012).

[23] E. Altman and R. Vosk, arXiv: 1408.2834 (2014).

[24] S. Trotzky et al., Nat. Phys. 8, 325 (2012).

[25] B. Paredes, F. Verstraete, and J. I. Cirac, Phys. Rev. Lett. 95, 140501 (2005).

[26] See Supplemental Material for exact results in free models, entanglement entropy, level statistics for XXZ and Bose-Hubbard models, and a description of the LCRG algorithm.

[27] P. Barmettler et al., Phys. Rev. Lett. 102, 130603 (2009); New J. Phys. 12, 055017 (2010).

[28] T. Enss and J. Sirker, New J. Phys. 14, 023008 (2012).

[29] D. Giuliano, D. Rossini, P. Sodano, and A. Trombettoni, Phys. Rev. B 87, 035104 (2013).

[30] T. Fukuhara et al., Nat. Phys. 9, 235 (2013).

[31] N. Y. Yao et al., arXiv:1311.7151 K. R. A. Hazzard et al., arXiv:1406.0937

[32] S. R. White, Phys. Rev. Lett. 69, 2863 (1992); A. J. Daley et al., J. Stat. Mech. P04005 (2004); S. R. White and A. E. Feiguin, Phys. Rev. Lett. 93, 076401 (2004).

[33] E. H. Lieb and D. W. Robinson, Commun. Math. Phys. 28, 251 (1972).

[34] F. Andraschko and J. Sirker, Phys. Rev. B 89, 125120 (2014).

[35] V. A. Yurovsky, and M. Olshanii, Phys. Rev. Lett. 106, 025303 (2011).

[36] N. Motoyama, H. Eisaki, and S. Uchida, Phys. Rev. Lett. 76, 3212 (1996).

[37] K. M. Kojima et al., Phys. Rev. B 70, 094402 (2004).

[38] J. Sirker et al., Phys. Rev. Lett. 98, 137205 (2007); J. Stat. Mech. P02015 (2008).

[39] T. Enss, F. Andraschko, and J. Sirker (2014) (to be published). 


\section{Supplemental Material: \\ "Purification and many-body localization in cold atomic gases" \\ by F. Andraschko, T. Enss, and J. Sirker}

We present in Sec. I additional information about the exact results for the free models, and in Sec. II data for the spin-only entanglement entropy for the $X X Z$ model. In Sec. III we discuss in detail the calculation of the level statistics for finite chains and in Sec. IV the LCRG algorithm.

\section{Free models}

The Bose-Hubbard model with $V=0$ reduces to free bosons for $U=0$ and to hard-core bosons for $U=\infty$. Similarly, the $X X Z$ chain for $\Delta=0$ reduces to free fermions.

\section{Difference in occupation of even and odd sites}

For the difference in occupation between the even and odd sites, $\Delta n(t)$, the statistics does not matter in the clean case, $D=0$, and in all three limits we find

$$
\Delta n^{D=0}(t)=J_{0}(2 J t) / 2
$$

where $J_{0}(t)$ is the Bessel function of the first kind which decays $\sim 1 / \sqrt{t}$ for large $t$. The statistics is also irrelevant for infinite disorder, $D=\infty$, where the chain splits into decoupled segments and the full time evolution of $\Delta n^{D=\infty}(t)$ can be calculated exactly as well. Using a Fourier representation one obtains

$$
\Delta n^{(\ell)}(t)=\frac{1}{2 \ell} \sum_{k=1}^{\ell} \exp \left[2 i J t \cos \left(\pi \frac{k}{\ell+1}\right)\right]
$$

for a segment of length $\ell$ with open boundary conditions and constant disorder potential. Here, the contributions with momenta $k$ and $\ell+1-k$ are complex conjugate, so that $\Delta n(t)$ is real. Initially, $\Delta n^{(\ell)}(t=0)=1 / 2$ for all segment lengths $\ell$, and for large segments $\Delta n^{(\ell \rightarrow \infty)}(t) \rightarrow \Delta n^{D=0}(t)$ approaches the thermodynamic limit result of the chain without disorder, Eq. (1). The time evolution of the whole chain is then given by summing up the time evolution of segments of varying length $\ell$, weighted by their probability of occurrence $p_{\ell}=\ell / 2^{\ell+1}$,

$$
\Delta n^{D=\infty}(t)=\sum_{\ell=1}^{\infty} p_{\ell} \Delta n^{(\ell)}(t)=\frac{1}{2}\left[\frac{1}{4} \times 1+\frac{2}{8} \times \cos (J t)+\frac{3}{16} \times(1+2 \cos (\sqrt{2} J t))+\cdots\right],
$$

where we have displayed $\Delta n^{(\ell)}(t)$ for open chains of lengths $\ell=1,2,3$ explicitly. The series converges exponentially fast.

\section{Entanglement entropy}

For the considered models with binary disorder, there are two kinds of entanglement entropies: one with and one without ancillas. Without ancillas, the Hilbert space of the system, consisting of spins or bosons, is written as a direct product, $\mathcal{H}=\mathcal{H}_{A} \otimes \mathcal{H}_{B}$, and the disorder-averaged entanglement entropy of block $A$ is defined as

$$
\overline{\mathcal{S}}_{\text {ent }}(t)=-\frac{1}{2^{N}} \sum_{\mathcal{C}} \operatorname{Tr}\left\{\rho_{A}^{\mathcal{C}}(t) \ln \rho_{A}^{\mathcal{C}}(t)\right\}
$$

with $\rho_{A}^{\mathcal{C}}(t)=\operatorname{Tr}_{B} \rho^{\mathcal{C}}(t)$. Here $\mathcal{C}$ denotes the $2^{N}$ configurations of the binary disorder potential for a chain of length $N$ and $\rho^{\mathcal{C}}(t)$ the corresponding density matrix. In the paper we have used an enlarged Hilbert space consisting of the system (s) and ancillas (a) with $\mathcal{H}=\mathcal{H}_{A_{s}} \otimes \mathcal{H}_{A_{a}} \otimes \mathcal{H}_{B_{s}} \otimes \mathcal{H}_{B_{a}}$ and the entanglement entropy shown in Fig. 2 and Fig. 5 of the paper is defined as

$$
S_{\text {ent }}(t)=-\operatorname{Tr}\left\{\rho_{A}(t) \ln \rho_{A}(t)\right\}
$$




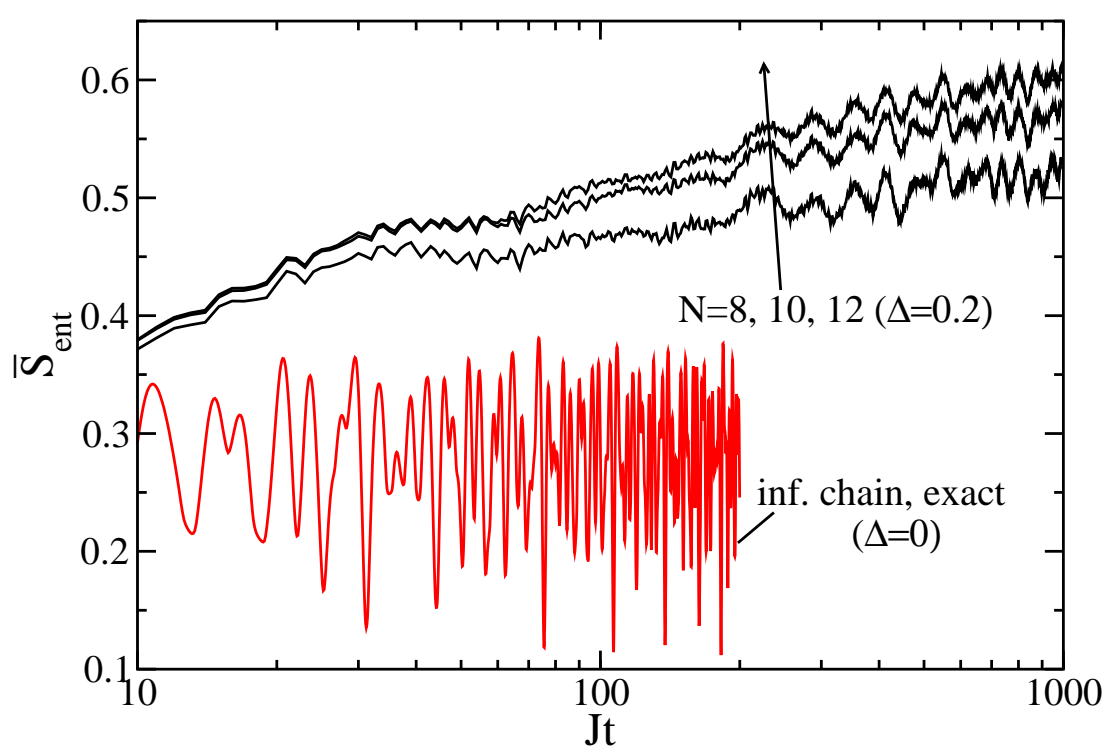

FIG. 6: Disorder-averaged entanglement entropy, Eq. (4), for the $X X Z$ chain with $D=4000, \Delta=0.2$, and system sizes $N=8,10,12$. For comparison, the exact result for an infinite $X X$ chain $(\Delta=0)$ with infinite disorder is shown-for clarity only up to $J t=200$ - where $\overline{\mathcal{S}}_{\text {ent }}(t)$ is bounded.

with $\rho_{A}(t)=\operatorname{Tr}_{B_{s}} \operatorname{Tr}_{B_{a}} \rho(t)$. Starting from a product state, the entanglement spreads during the time evolution via the hopping (spin flips) on the system sites, while the ancillas do not have any dynamics. The asymptotic functional time dependence of both entanglement entropies will therefore be the same.

For the non-interacting cases, the time evolution of the disorder averaged entanglement entropy $\overline{\mathcal{S}}_{\text {ent }}(t)$ can be calculated analytically for infinite disorder. The probability that a chain segment with length $\ell \geq 2$ is cut with $d$ sites left open and $\ell-d \geq 1$ sites traced over is $1 / 2^{\ell+1}$. There are $\ell-1$ possibilities to cut a segment of length $\ell$ and

$$
\sum_{\ell=2}^{\infty} \frac{\ell-1}{2^{\ell+1}}=\frac{1}{2}
$$

while with probability $1 / 2$ the cut is in between segments in which case the entanglement entropy is zero. The disorder averaged time-dependent entanglement entropy is then given by

$$
\overline{\mathcal{S}}_{\text {ent }}(t)=\sum_{\ell=2}^{\infty} \sum_{d=1}^{\ell-1} \frac{\mathcal{S}_{\text {ent }}^{(d, \ell)}(t)}{2^{\ell+1}} \leq \sum_{\ell=2}^{\infty} \sum_{d=1}^{\ell-1} \frac{\ln \Gamma_{d}}{2^{\ell+1}} .
$$

Here $\mathcal{S}_{\text {ent }}^{(d, \ell)}(t)$ is the entanglement entropy of a block of length $d$ obtained by tracing out $d-\ell$ sites in a segment of length $\ell$ and $\Gamma_{d}$ is the dimension of the reduced density matrix of this block. For the $X X$ and the hard-core boson case, in particular, we have $\Gamma_{d}=2^{d}$ leading to the upper bound $\overline{\mathcal{S}}_{\text {ent }}(t) \leq \ln 2$. A similar bound also exists for the Bose-Hubbard model in the general case.

Within the purification approach used in the paper for the LCRG calculations, the time-evolved state is a superposition of states for each disorder configuration, i.e. in the non-interacting cases at infinite disorder, different segment lengths are participating in the superposition with the appropriate weight. Since states containing long segments are exponentially suppressed, the entanglement entropy with ancillas, $S_{\text {ent }}$, shown for the $X X$ case in the inset of Fig. 2 in the paper, is still bounded. The existence of a bound can be shown analytically in this case as well.

\section{Spin-only entanglement entropy for the $X X Z$ model}

To show that the two entanglement entropies defined in Eq. (4) and Eq. (5), respectively, have the same functional dependence on $t$ for the $X X Z$ model we present in Fig. 6 the spin-only entanglement entropy defined in Eq. (4) - which cannot easily be obtained using LCRG - for finite systems of size $N=8,10,12$ where the disorder average has been performed exactly. To make the dependence $\overline{\mathcal{S}}_{\text {ent }} \sim \ln t$ at long times clearly visible, the data are calculated with a time step of $\delta t=1$ and a subsequent moving average with range 10 is performed. 

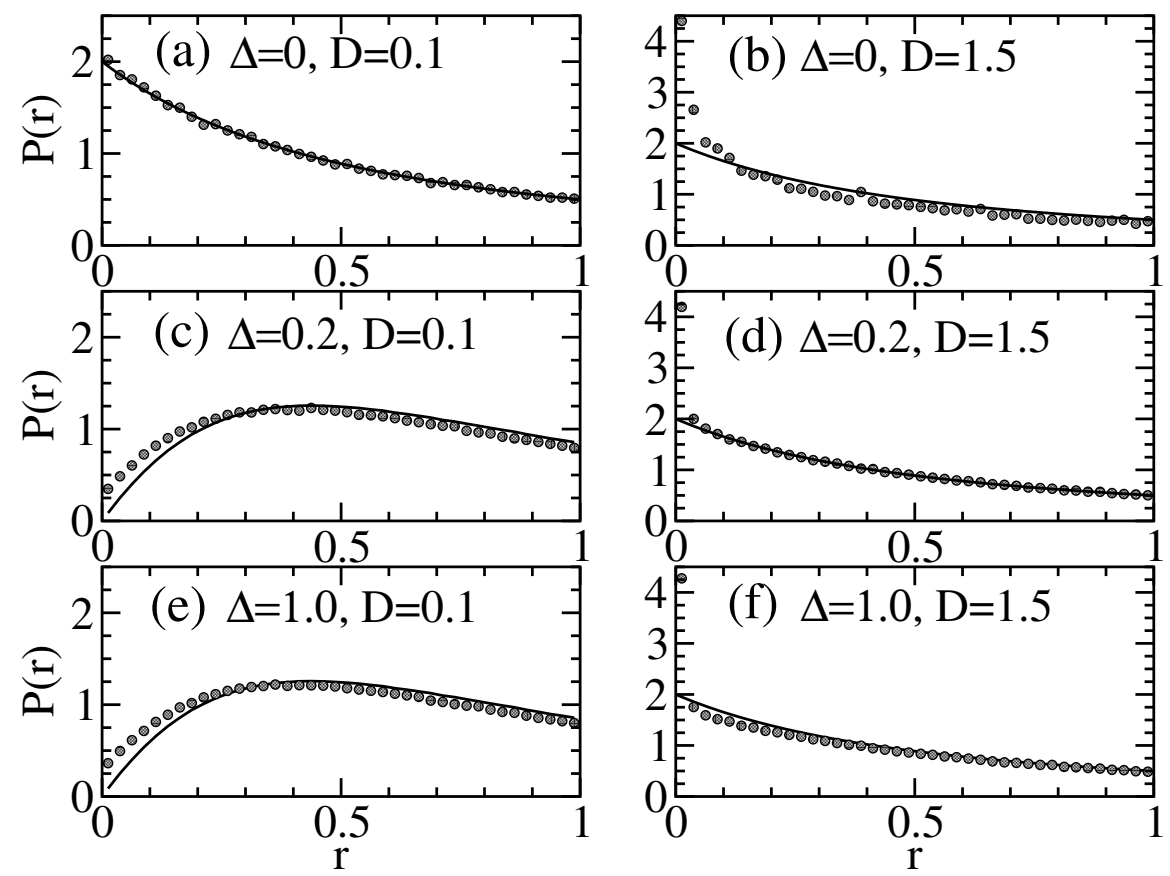

FIG. 7: Gap distribution function $P(r)$ for $X X Z$ chains with $N=14$ sites (symbols). Lines represent $P(r)=2 /(1+r)^{2}$ in the Poissonian case, or the result from diagonalizing random matrices in the GOE case. (a,b) In the non-interacting case, $\Delta=0$, the level statistics is Poissonian for all disorder strengths. (c,d) In the weakly interacting case, the level statistics changes from GOE at small disorder to Poissonian at intermediate disorder. (e,f) A change from GOE to Poissonian statistics is also observed in the strongly interacting case.
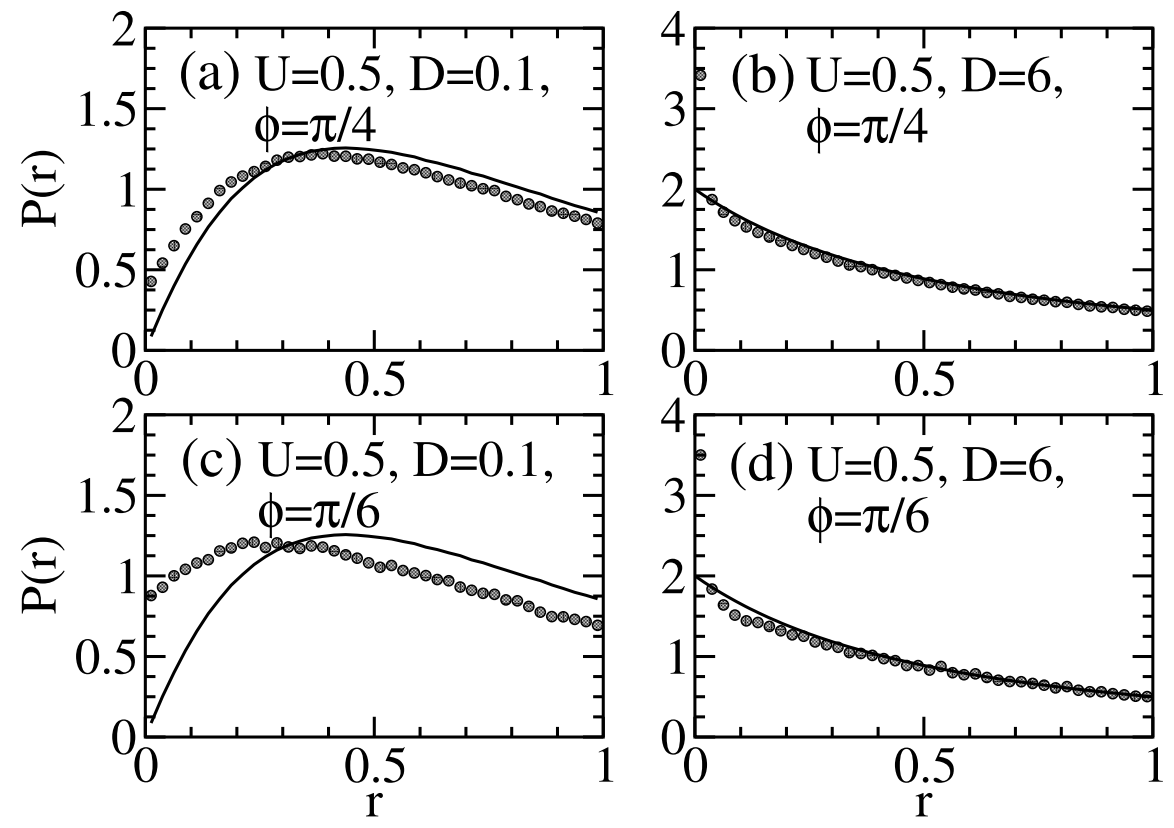

FIG. 8: Gap distribution function $P(r)$ for Bose-Hubbard chains with $V=0$ and $N=12$ sites (symbols). Lines represent the theoretically expected distributions. The level statistics changes from GOE for weak disorder, panel (a,c), to Poissonian for intermediate disorder, panel $(\mathrm{b}, \mathrm{d})$. We consider two filling fractions for the immobile bosons parametrized by $\phi$, see text. 


\section{Level statistics}

The existence of an MBL phase for the Bose-Hubbard and the $X X Z$ model with binary disorder is supported by the level statistics for finite chains. In the paper we have been following the approach in Refs. 11, 12 , by considering the ratio $0 \leq r=\min \left\{\delta_{n}, \delta_{n-1}\right\} / \max \left\{\delta_{n}, \delta_{n-1}\right\} \leq 1$ between two consecutive gaps of adjacent energy levels, $\delta_{n}=E_{n+1}-E_{n} \geq 0$. If a set of local or quasi-local conserved charges exists which allows to uniquely label the eigenvalues then the level statistics is uncorrelated and the distribution function of gap ratios $r$ is given by $P(r)=2 /(1+r)^{2}$. We expect this to be the case if the model is integrable and when the model is in an MBL phase. For a generic non-integrable model with extended states, on the other hand, the level statistics is expected to follow a Gaussian orthogonal ensemble (GOE). In this case, the distribution function of gap ratios $P(r)$ is not known analytically. As in Ref. [11] we determine $P(r)$ by exactly diagonalizing 10000 random $(3432 \times 3432)$ GOE matrices.

\section{$X X Z$ model}

The $X X Z$ chain is integrable in the clean case leading to a Poissonian level statistics. As shown in Fig. 7(a,b) the level statistics remains Poissonian also with disorder in the $X X$ case, $\Delta=0$, where the model is equivalent to free spinless fermions.

For finite interaction strength, on the other hand, we find a GOE statistics for weak disorder and a Poissonian statistics for intermediate disorder, see Fig. 7( c,d), and Fig. 7(e,f). In the intermediate disorder case, the Poissonian statistics is already clearly visible which means that the localization length is smaller than the system size, strongly supporting the existence of an MBL phase. For the weakly disordered case, shown in Fig. 7(c) and Fig. 7(e), there are two possibilities: the system either has extended states or the localization length is much larger than the considered system size. Finding out which one of the two scenarios is true is not easily possible based on finite size data and is beyond the scope of this letter. The crossover from a Poissonian level statistics in the clean integrable case to a GOE statistics at weak disorder can also be understood as the onset of chaos which might remain incomplete because of the formation of new quasi-local charges [35, see also Fig. 4 in the main paper. The deviations in Fig. 7(b,d,f) near $r \approx 0$ from the result expected for a Poissonian statistics are finite size effects and become smaller with increasing system size.

\section{Bose-Hubbard model with $V=0$}

Finally, we present additional supporting data for the level statistics of the Bose-Hubbard model with $V=0$ in Fig. 8. The Bose-Hubbard model is not integrable so that the level statistics is GOE without disorder. For small disorder we still find agreement with GOE statistics, see Fig. 8, which means, in analogy to the $X X Z$ case, that the states either remain extended or that the localization length for this disorder strength is much larger than the system size. For disorder strength $D=6$ shown in Fig. $8(\mathrm{~b})$, on the other hand, the level statistics is Poissonian and the system in an MBL phase.

\section{Details on the LCRG algorithm}

We study the dynamics of the disordered $X X Z$ and Bose-Hubbard models with the LCRG algorithm [28. This variant of time-dependent DMRG makes use of the light cone structure inherent to the Trotter-Suzuki decomposition of the time evolution operator, as shown in Fig. 9. If the Trotter step is chosen small enough with respect to the Lieb-Robinson velocity of the system, simulating the depicted light cone is sufficient to calculate observables in the thermodynamic limit accurately. To simulate binary disorder, we follow the purification approach introduced in Ref. 25: to each lattice site we add an ancilla spin-1/2. The ancilla degrees of freedom are coupled to the physical system by adding to the Hamiltonian $H^{d i s}=-2 J D \sum_{j} s_{j}^{z} s_{j, a n c}^{z}$ for the $X X Z$ model, and $H^{d i s}=J D \sum_{j} n_{j}^{A} n_{j}^{B}$ for

the Bose-Hubbard model, where $n_{j}^{A}\left(n_{j}^{B}\right)$ is the particle number operator for the mobile (immobile) bosons. For binary disorder, we prepare the ancilla spins (immobile bosons) in the initial product state $\prod_{j}\left(|\uparrow\rangle_{j}+|\downarrow\rangle_{j}\right) / \sqrt{2}$ or $\prod_{j}\left(|1\rangle_{j}+|0\rangle_{j}\right) / \sqrt{2}$, respectively, so they represent a uniform superposition of all possible realizations of static binary disorder. We note that it is possible to prepare the immobile bosons in the more general state $\prod_{j}\left(\sin \phi|1\rangle_{j}+\cos \phi|0\rangle_{j}\right)$, for any $\phi \in[0,2 \pi)$, instead, realizing a weighted superposition of binary disorder. Here, $\sin ^{2} \phi$ determines the average 

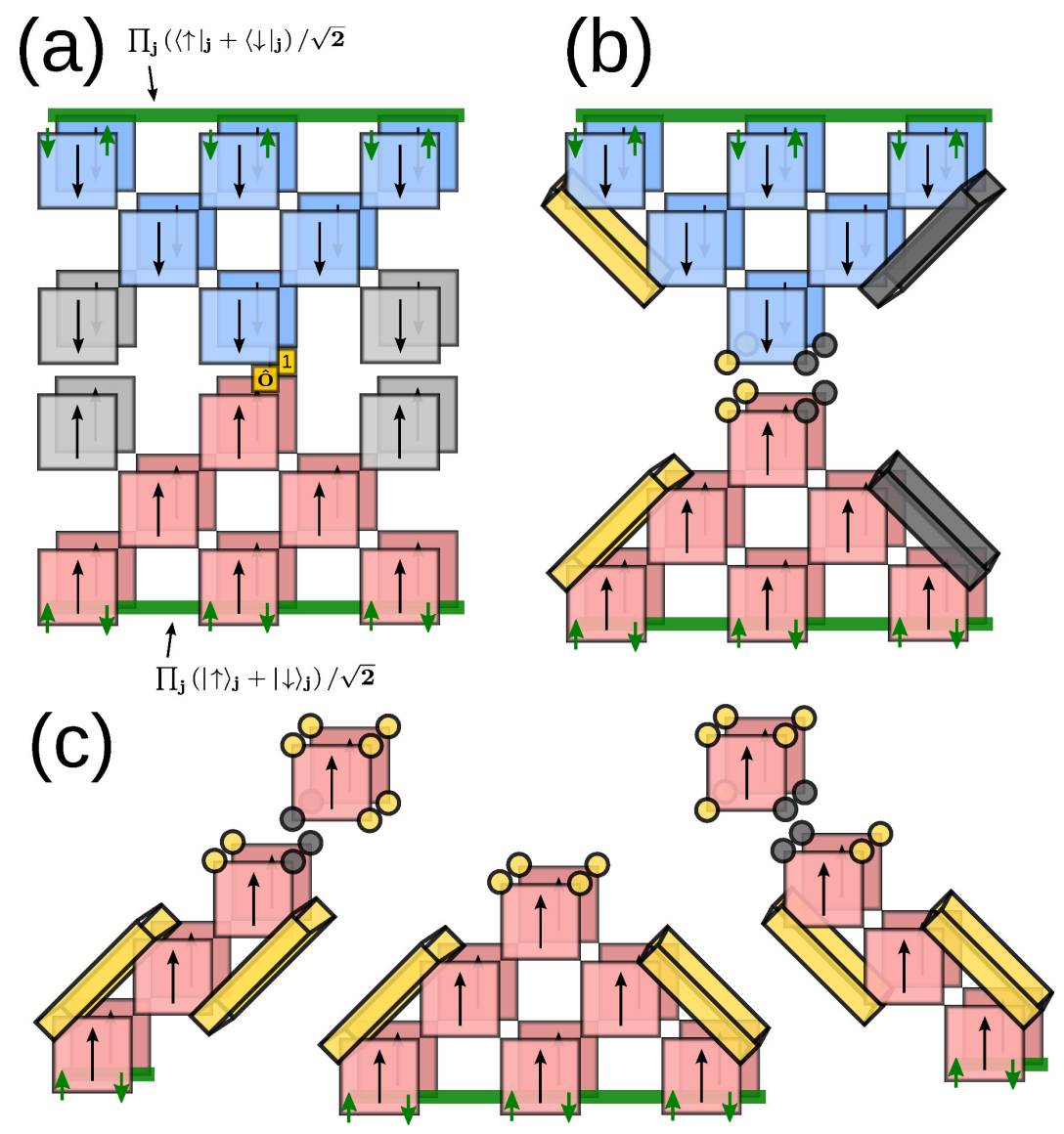

FIG. 9: LCRG algorithm with exact disorder average for the $X X Z$ model: (a) Measurement of a one-point function $\langle\hat{O}(t)\rangle$. The grey plaquettes cancel out due to unitarity. (b) Reduced density matrix for renormalization. Grey indices are summed over. Circles denote single spin or ancilla indices, boxes the block indices of spins and ancillas. (c) Growth step of left and right transfer matrices, and central light cone.

density $\bar{n}^{B}$ of the immobile boson species in an experiment. While a thorough analysis of the stability of the MBL phase with respect to $\bar{n}^{B}$ is outside the scope of this work, we present a comparison of the level statistics the BoseHubbard model for average densities $\bar{n}^{B}=1 / 2(\phi=\pi / 4)$ and $\bar{n}^{B}=1 / 4(\phi=\pi / 6)$ in Fig. 8 . The data demonstrate that the MBL phase is stable against a variation in the density of the immobile bosons.

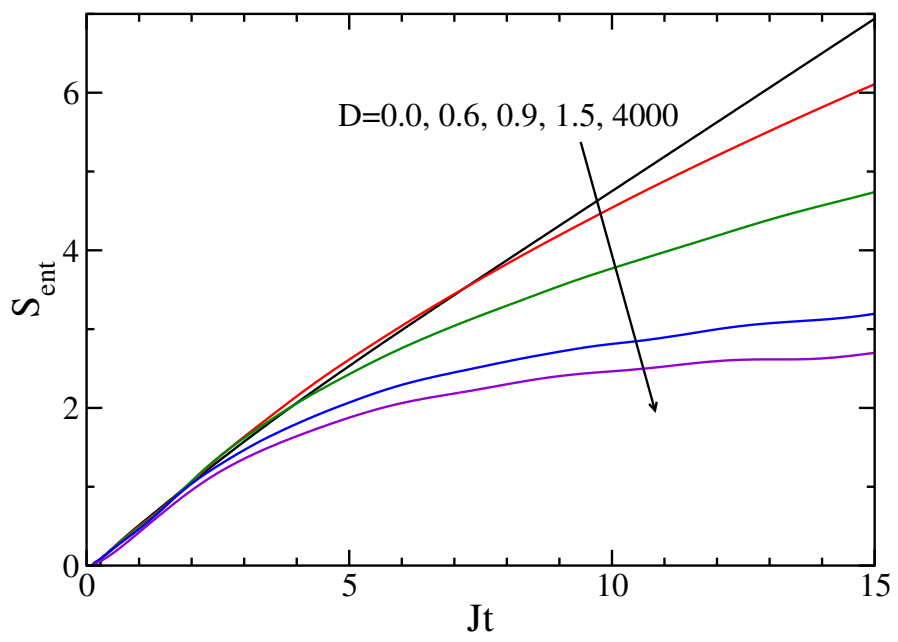

FIG. 10: Entanglement entropy for the $X X Z$ model with $\Delta=1$. 
Details of the LCRG algorithm with ancillas are shown in Fig.9 in each step, the left and right transfer matrices are grown by adding a single Trotter plaquette (Fig. 9c). Then, the central light cone is alternatingly grown to the left and right by contracting it with the corresponding transfer matrix. Subsequently, a standard DMRG renormalization is performed on the reduced density matrix depicted in Fig. 9b. A defining feature for the MBL phase is the logarithmic growth of the entanglement entropy, $S_{\text {ent }} \propto \ln t$. This makes it possible to simulate the disordered system for times up to an order of magnitude longer than the clean system, in spite of having doubled the local Hilbert space dimension. In Fig. 10, we show the gradual improvement of the scaling of the entanglement entropy with increasing disorder strength on the example of the Heisenberg chain. 